OPEN ACCESS

Edited by:

Musa R. Khaitov,

Institute of Immunology, Russia

Reviewed by:

Ourania Tsitsilonis,

National and Kapodistrian University of

Athens, Greece

Pramod Kumar Srivastava,

University of Connecticut,

United States

*Correspondence:

Chao Cheng

chao.cheng@bcm.edu

Cheng-Chi Chao

chengchi@biomap.com

Specialty section:

This article was submitted to Antigen Presenting Cell Biology,

a section of the journal

Frontiers in Immunology

Received: 30 April 2021 Accepted: 16 December 2021

Published: 17 January 2022

Citation:

Jiang $C$, Schaafsma $E$

Hong W, Zhao Y, Zhu K,

Chao C-C and Cheng C (2022)

Influence of T Cell-Mediated Immune

Surveillance on Somatic Mutation

Occurrences in Melanoma.

Front. Immunol. 12:703821.

doi: 10.3389/fimmu.2021.703821

\section{Influence of T Cell-Mediated Immune Surveillance on Somatic Mutation Occurrences in Melanoma}

\author{
Chongming Jiang ${ }^{1}$, Evelien Schaafsma ${ }^{2}$, Wei Hong ${ }^{1}$, Yanding Zhao ${ }^{1}$, Ken Zhu ${ }^{3}$, \\ Cheng-Chi Chao ${ }^{4 *}$ and Chao Cheng ${ }^{1,5,6,7 *}$
}

\begin{abstract}
${ }^{1}$ Department of Medicine, Baylor College of Medicine, Houston, TX, United States, ${ }^{2}$ Department of Molecular and Systems Biology, Dartmouth College, Hanover, NH, United States, ${ }^{3}$ Medical School, UT Southwestern Medical Center, Dallas, TX, United States, ${ }^{4}$ Antibody Discovery, Chempartner Corporation, South San Francisco, CA, United States, ${ }^{5}$ Dan L. Duncan Comprehensive Cancer Center, Baylor College of Medicine, Houston, TX. United States, ${ }^{6}$ Department of Biomedical Data Science, Geisel School of Medicine at Dartmouth, Lebanon, NH, United States, ${ }^{7}$ The Institute for Clinical and Translational Research, Baylor College of Medicine, Houston, TX, United States
\end{abstract}

Background: Neoantigens are presented on the cancer cell surface by peptide-restricted human leukocyte antigen (HLA) proteins and can subsequently activate cognate $T$ cells. It has been hypothesized that the observed somatic mutations in tumors are shaped by immunosurveillance.

Methods: We investigated all somatic mutations identified in The Cancer Genome Atlas (TCGA) Skin Cutaneous Melanoma (SKCM) samples. By applying a computational algorithm, we calculated the binding affinity of the resulting neo-peptides and their corresponding wild-type peptides with the major histocompatibility complex (MHC) Class I complex. We then examined the relationship between binding affinity alterations and mutation frequency.

Results: Our results show that neoantigens derived from recurrent mutations tend to have lower binding affinities with the MHC Class I complex compared to peptides from non-recurrent mutations. Tumor samples harboring recurrent SKCM mutations exhibited lower immune infiltration levels, indicating a relatively colder immune microenvironment.

Conclusions: These results suggested that the occurrences of somatic mutations in melanoma have been shaped by immunosurveillance. Mutations that lead to neoantigens with high $\mathrm{MHC}$ class I binding affinity are more likely to be eliminated and thus are less likely to be present in tumors.

Keywords: Melanoma, recurrent mutation, immunosurveillance, neoantigen, antigen presentation

\section{INTRODUCTION}

Cancer is a genetic disease caused by genomic abnormalities including somatic mutations, which result in mutated antigens (i.e., neoantigens). Neoantigens derived from non-synonymous mutations can be recognized, bound, and presented on the tumor cell surface by major histocompatibility complex (MHC) proteins. $\mathrm{T}$ cells can recognize and attack tumor cells 
presenting these neoantigens, which is known as T cell-mediated cancer immunosurveillance $(1,2)$. However, tumors can develop different strategies to avoid recognition and elimination by the immune system (3-5).

Evolving neoplasms accumulate non-synonymous mutations at a high rate, leading to the expression of antigenic epitopes that might be recognized by the immune system (6). According to the theory of immunosurveillance, a functional immune system can recognize and eliminate tumor cells harboring antigenic mutations $(1,7)$. Tumor cells presenting immune-activating neoantigens are more likely to be eliminated through $\mathrm{T}$-cell recognition as compared to non-immunogenic mutations (810). Consequently, somatic mutations abrogating essential immune functions (e.g., mutations in B2M and HLA genes) are generally positively selected for in tumors and are common in different types of cancers (11). In contrast, the majority of somatic mutations are under negative selection during the tumorigenesis by immunosurveillance. A number of previous studies have shown that the immune system can exert strong selection pressure on neoantigens in both untreated and treated tumors (12-15). During lung cancer evolution, the immune system also exhibits neoantigen-editing in which cells with immunogenic mutations are eliminated (14). Indeed, the observed counts of neoantigens were unexpectedly low in some tumor types $(16,17)$, suggesting the impact of negative selection posed by immunosurveillance. However, other studies have suggested that neoantigen selection by the immune system becomes negligible in untreated tumor samples when considering mutational signatures $(18,19)$.

The potential impact of immunosurveillance on gene mutation abundance is determined by multiple factors, namely, the category of mutated genes (cancer driver or passenger genes), mutation frequency $(12,20-22)$, the expression level of the mutated genes (23), and HLA functionality (proficient or deficient) (24). The capacity of antigens to induce a $\mathrm{CD}^{+} \mathrm{T}$ cell immune response (antigenicity) is mainly determined by their binding affinity with the MHC class I (MHC-I) complex. In this study, we investigated the non-synonymous somatic mutations identified in the TCGA Skin Cutaneous Melanoma (SKCM) samples. We applied a computational pipeline to calculate the binding affinity of the resulting neoantigens with the MHC-I complex. As a control, we calculated the MHC-I binding affinity of the wide-type peptide. We found that neoantigens of recurrent mutations have significantly lower MHC-I binding affinity than those from non-recurrent mutations. These results were observed for somatic mutations presenting in both melanoma-specific driver and passenger genes. In addition, we investigated the potential influence of HLA genotypes and HLA gene deficiency (mutation and loss events). Our results suggested that the somatic mutation landscape in melanoma is shaped by $\mathrm{T}$ cell-mediated immunosurveillance.

\footnotetext{
Abbreviations: MHC, major histocompatibility complex; MHC-I, MHC class I; HLA, human leukocyte antigen; dRM, recurrent mutation in driver genes; dNRM, non-recurrent mutation in driver genes, $\mathrm{pRM}$, recurrent mutation in passenger genes; pNRM, non-recurrent mutation in passenger genes; WES, whole-exome sequencing); GDC, Genomic Data Commons; ROC, Receiver Operating Characteristics; AUC, Area Under The Curve.
}

\section{RESULTS}

\section{Quantification of the Effect of Non- Synonymous Mutations on MHC-I Binding Affinity}

To investigate how $\mathrm{T}$ cell-mediated immunosurveillance shapes the mutational landscape in SKCM tumors, we calculated a residuecentric presentation score to quantify MHC-I binding affinity changes that resulted from non-synonymous mutations using the method described in Figure 1. First, for each non-synonymous mutation, we enumerated nine 9-mers (peptides with 9 amino acids) that overlap with the mutated residue (Figure 1A). We applied NetMHCPan4.0 (25) to these 9-mers to calculate their binding affinity with the MHC-I complex (Figure 1B) using six patient-specific HLA class I alleles (two HLA-A, two HLA-B, and two HLA-C genes). We decided on NetMHCPan4.0 for MHC-I binding affinity calculations due to its overall high and stable performance as demonstrated in previous studies (28-30). Each mutation-derived peptide received a prediction score within $[0,1]$, with higher scores indicating higher binding affinities with the MHC-I complex. In total, 54 binding scores were calculated for the nine peptides that resulted from a mutation with the six HLA class I alleles. Second, we calculated the maximum value for each HLA allele to capture the peptide with the highest binding affinity, resulting in six HLA class I allele-specific affinity scores. Finally, we selected the maximum value of the six HLA class I allele-specific affinity scores to represent the best MHC-I complex presentation potential for peptides derived from a specific non-synonymous mutation (see Materials and Methods section). In Figure 1C, we evaluated the prediction accuracy of this pipeline by applying it to a combined benchmark data (Supplementary Table 1), containing experimentally identified MHC-I binding peptides (27) and nonbinding control peptides (26). As shown, our pipeline can accurately classify these two types of peptides with an average AUC (area under receiver operating characteristic curve) score of 0.88 (Figure 1C). After verifying the robustness of the pipeline, we applied it to a total of 122,603 non-synonymous mutations identified from 345 TCGA SKCM tumor samples (Supplementary Table 2).

\section{Neoantigens Derived From Recurrent Mutations Have Lower Binding Affinities Than Those From Non-Recurrent Mutations}

We hypothesize that somatic mutations that result in neoantigen with high MHC-I binding affinity are subject to negative selection via immunosurveillance and are therefore less likely to be present at high frequencies in tumor samples. To test this hypothesis, we divided non-synonymous (NS) mutations identified in the TCGA SKCM samples (29) into recurrent (occurred in at least three samples) and non-recurrent mutations (occurred in only one sample) (Figure 2A). In total, we identified 5,677 recurrent and 97,618 non-recurrent NS mutations. For each mutation, we calculated the aforementioned binding score that indicates the putative highest possible affinity score a mutation-derived peptide can receive given the HLA-A, B, and C allele type of a patient (see 
A

Non-synonymous mutation

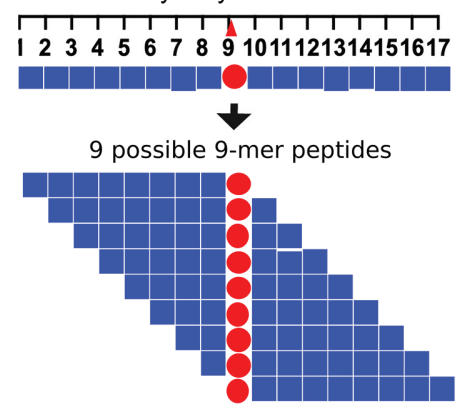

C

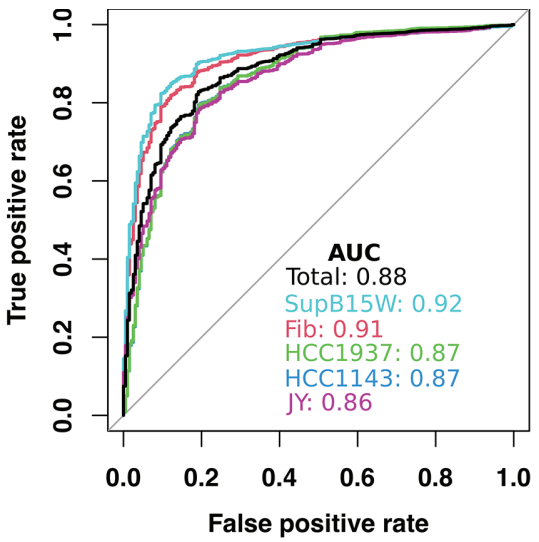

B

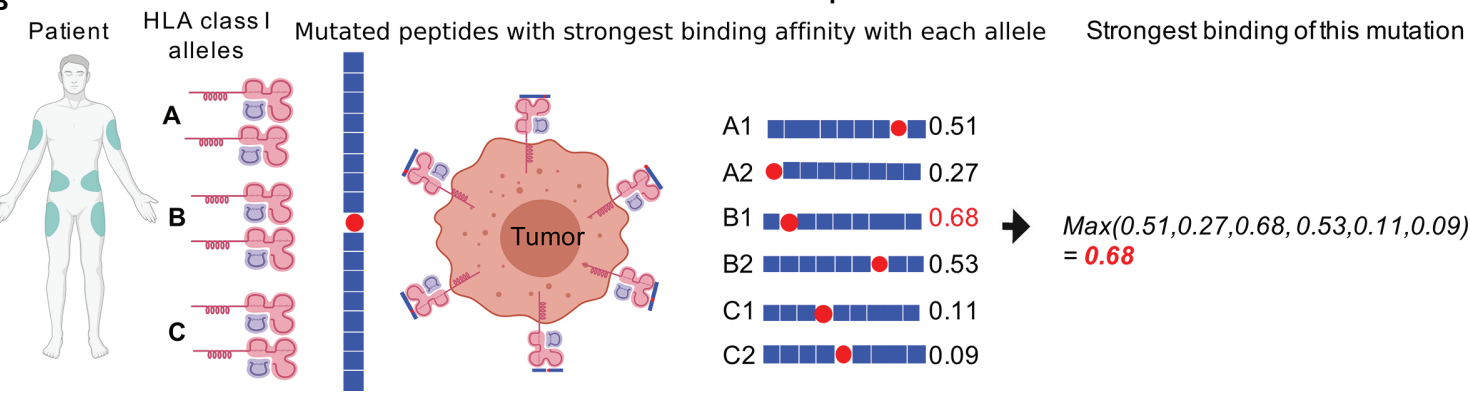

FIGURE 1 | Calculation of the MHC-I binding affinity of the neoantigen derived from a non-synonymous mutation. (A) For each mutation, a total of 17 amino acids centering at the mutated site were considered. The binding affinities with MHC-I complex of the nine possible 9-mer peptides were calculated by using NetMHCPan4.0 (25). (B) During the MHC-I binding affinity calculation, patient-specific HLA genotypes were used. Each patient has two alleles for HLA-A, B, and C gene, resulting in six total alleles. For each allele, MHC-I binding affinities to the nine 9-mers were calculated. The final MHC-I binding affinity of the somatic mutation was calculated as the maximum value of the 9 (peptides) $\times 6$ (alleles) binding scores. Figure created using BioRender (https://biorender.com/). (C) The performance of our MHC-I binding affinity pipeline was evaluated by classifying MHC binding and non-MHC binding epitopes in experimental data from previous studies (26, 27). The data provided peptide sequences for a collection of MHC binding and non-MHC binding epitopes in 5 different human cell lines (Fibroblast, SupB15W, JY, HCC1937, and HCC1143).

Materials and Methods for details). First, we calculated MHC-I binding affinity correlations between mutation-derived peptides and the corresponding wild-type peptides. Overall, we observed fairly high correlations with recurrent mutations showing slightly lower correlations than non-recurrent mutations ( $\mathrm{r}=$ 0.70 versus 0.75 ) (Figure 2 B), indicating larger differences in MHC class I binding affinities upon mutation in recurrent mutations as compared to non-recurrent mutations. To quantify the effect of mutations on binding affinity, we calculated binding score differences between mutant and wildtype peptides to obtain $\Delta S$ for each mutation. The $\Delta S$ takes a value within $[-1,1]$ with the distribution shown in Figure 2C.

As shown in Figure 2D, recurrent mutations showed significantly lower binding affinity with MHC-I complex in their mutation-derived peptides than non-recurrent mutations (adjust P-value $\left(P_{a d j}\right)=1.2 \mathrm{e}-13$ ). We also compared the MHC-I binding scores of the corresponding wild-type peptides and found that the recurrent mutations showed significantly higher MHC-I binding scores than non-recurrent mutations $\left(P_{a d j}=\right.$ 0.0058). When $\Delta S$ values were compared, the recurrent mutations are significantly lower than non-recurrent mutations $\left(P_{a d j}=4.5 \mathrm{e}-42\right)$. Altogether, our results suggest that the presence of somatic mutations are likely shaped by immunosurveillance- tumor cells hosting mutations with higher MHC-I binding affinity are more likely to be eliminated and, as a consequence, these mutations are less to be present in tumors.

In addition to melanoma, we also applied the same analysis in 16 TCGA cancer types with available HLA genotypes and large sample size. Out of them, we found significantly $\Delta S$ reduction in recurrent (occurrence $\geq 3$ ) than non-recurrent mutations (occurrence $=1$ ) in 6 cancer types, breast cancer, cervical cancer, rectum adenocarcinoma, stomach adenocarcinoma, endometrial cancer, and kidney cancer (Figure S1). Some of the non-significant cancer types have relative low tumor mutation burden and therefore the numbers of recurrent mutations are small, which limited the statistical power for $\Delta \mathrm{S}$ comparison.

\section{Somatic Mutations in Driver and Passenger Genes}

Based on the functional impact of somatic mutations, genes may be categorized into cancer driver genes and passenger genes (31). Mutations in driver genes are more likely to confer growth advantages to tumor cells and are therefore often positively selected for during cancer development $(21,32)$. In this study, we utilized the 1,161 melanoma driver genes (DGs) reported by 
A

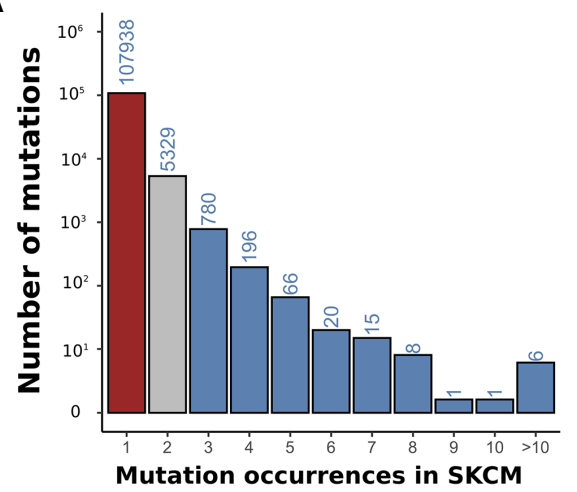

C

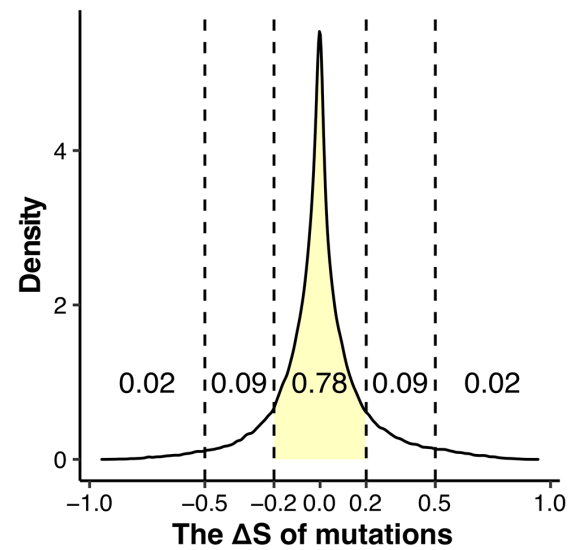

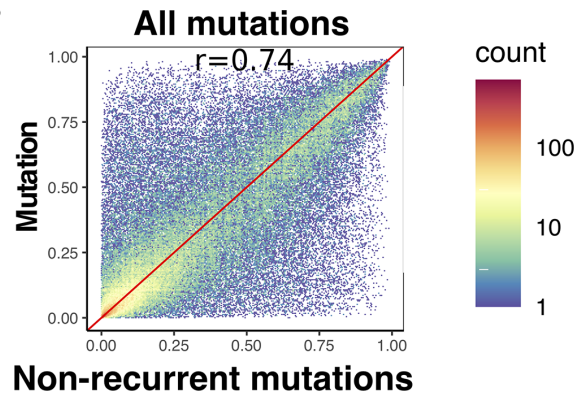

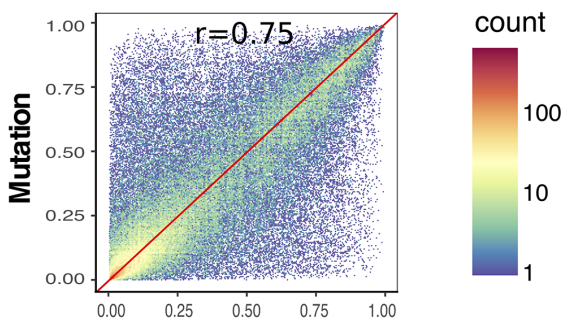

Recurrent mutations

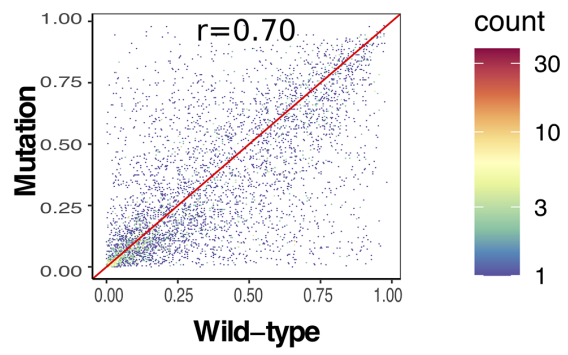

D

Mutation

Wild-type

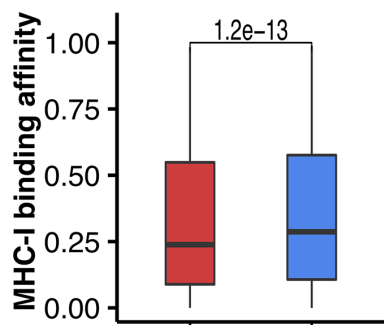

Recurrent Non-recurrent
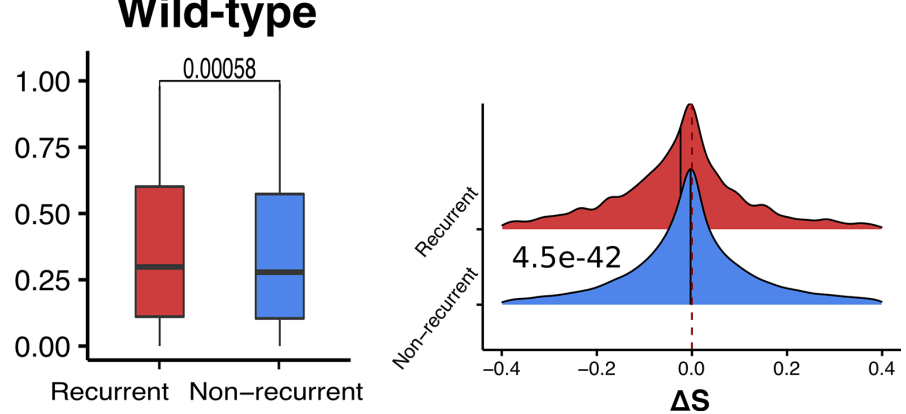

FIGURE 2 | Recurrent somatic mutations have significantly lower $\Delta$ S than non-recurrent mutations in SKCM. (A) The distribution of occurrences of non-synonymous mutations in SKCM. Mutation count was calculated as the number of melanoma patients harboring a specific somatic mutation. Based on the distribution, we defined recurrent mutations (blue) as those presenting in at least three melanoma samples, and non-recurrent mutations (red) as those presenting in only one sample. (B) Correlations between antigens derived from mutations (all, non-recurrent, and recurrent) and the corresponding wide-type in MHC-I binding affinities. (C) The density plot of $\Delta \mathrm{S}$, which was defined as the difference between the MHC-I binding score of mutant and wild-type antigens. (D) Comparison between recurrent vs non-recurrent mutations in the $\mathrm{MHC}$-I binding affinity with antigens derived from mutations (left) and the wild-type (middle), as well their difference ( $\Delta S$ ) (right). In all plots, P-values were calculated using two-sided Wilcoxon rank-sum test and adjusted for multiple testing by the Holm-Bonferroni method.

Chung et al. (33) as SKCM DGs. The distributions of NS somatic mutations in DGs and passenger genes (PGs) are shown in Figure 3A. We detected no significant differences in MHC-I binding scores between mutations in these two gene groups (Figure 3B and Figure S2A).
By further considering the frequency of mutations in the TCGA SKCM samples, we divided the DG and PG mutations into four groups: recurrent driver mutations (dRM), nonrecurrent driver mutations (dNRM), recurrent passenger mutations ( $\mathrm{pRM})$, and non-recurrent passenger mutations 
A

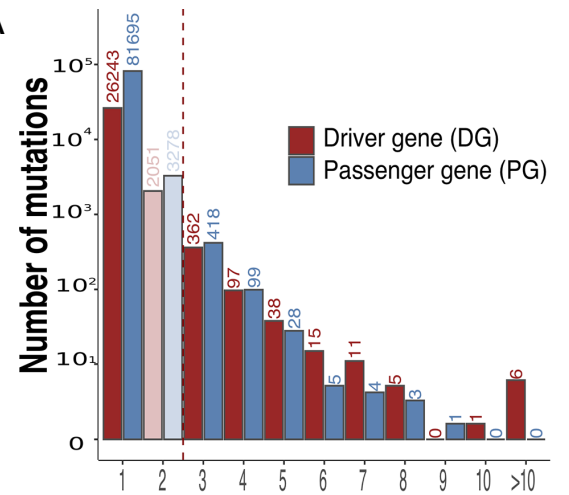

Mutation occurrences in SKCM
B

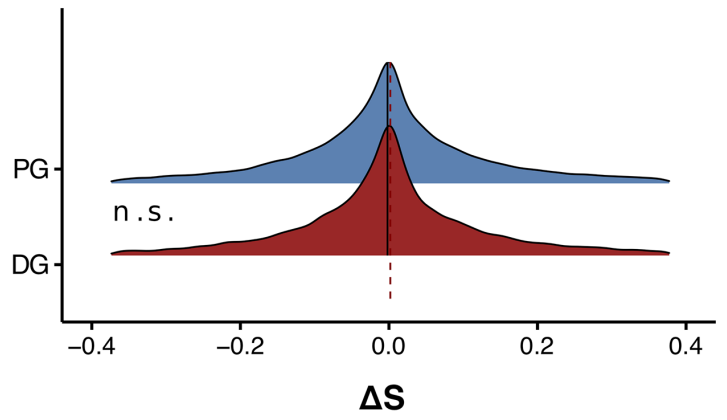

C

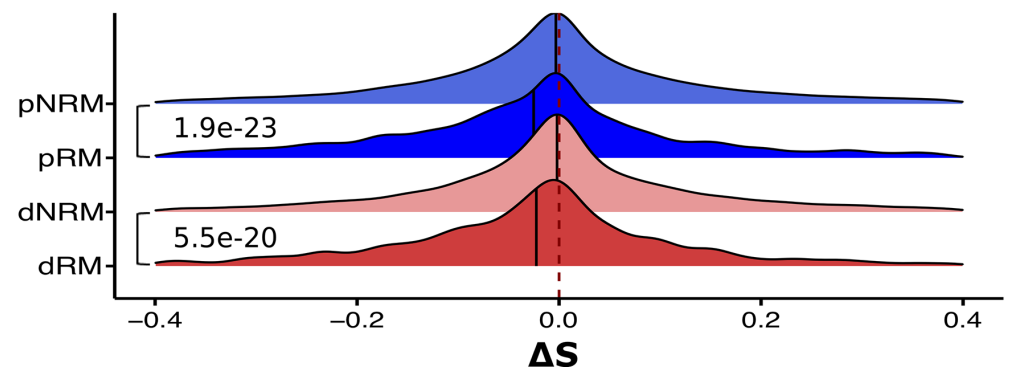

FIGURE 3 | Recurrent mutations have significantly lower $\Delta$ S than non-recurrent mutations in both driver and passenger genes. (A) The distribution of occurrences of non-synonymous mutations in melanoma samples. Mutations presenting in driver and passenger genes are separated. (B) No significant $\Delta$ S difference between somatic mutations in driver and passenger genes. n.s., not significant. (C) Comparison of $\Delta$ S between recurrent and non-recurrent mutations in driver and passenger mutations. dRM, recurrent mutation in driver genes; dNRM, non-recurrent in driver genes; pRM, recurrent mutation in passenger genes; pNRM, non-recurrent in passenger genes. P-values were calculated using two-sided Wilcoxon rank-sum tests.

(pNRM). A comparison between these groups indicated that in both driver and passenger genes, recurrent mutations showed significantly lower $\Delta S$ than non-recurrent mutations (Figure 3C and Figure S2B). Thus, neoantigens derived from recurrent mutations tend to have lower MHC-I binding affinities with respect to peptides from their wild-type counterparts.

\section{The Effect of Somatic Mutations and Loss of HLA Genes}

Previous studies have reported that the loss of HLA genes can affect tumor cell immune escape in lung cancer $(24,34)$. We thus examined the potential effect of HLA gene defects (HLA gene mutation or loss) on MHC-I binding affinity of neoantigens derived from somatic mutations in SKCM. We obtained the HLA gene mutation information of the TCGA melanoma samples from Castro et al. (11), and HLA gene loss information from Taylor et al. (35). In melanoma samples with HLA gene deficiency, the distributions of MHC-I binding affinities for dRMs, dNRMs, pRM, and pNRMs were similar to those with proficient HLA genes (Figures 4A, B). Significance was generally not reached when comparing the effect of HLA mutations
(Figures 4A, B), presumably due to reduced sample size (the number of samples with HLA gene mutation or loss is small).

\section{Melanoma Samples Harboring Recurrent Somatic Mutations Have Lower Immune and Stromal Scores}

Based on the number of recurrent mutations in each sample, we divided melanoma samples into two groups. The first group contained 215 melanoma samples with at least seven recurrent somatic mutations, while the second group contained 130 samples with less than seven recurrent mutations. We compared their immune microenvironment difference by calculating samplespecific immune scores and stromal scores based on their gene expression profiles using the ESTIMATE algorithm (36). These two scores indicated the relative abundance of infiltrating immune cells and stromal cells in the tumor samples, respectively. We found that melanoma samples harboring more recurrent mutations had significantly lower immune scores compared to those with less than 7 recurrent mutations $\left(P_{a d j}=0.0037\right)$. This was observed in both primary and metastatic melanoma samples $\left(P_{a d j}=0.0015\right.$, and 0.023, respectively), as shown in Figure 5A. Similarly, stromal 


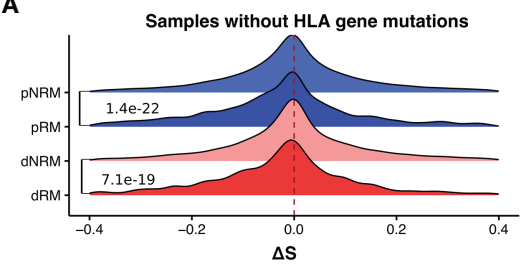

B

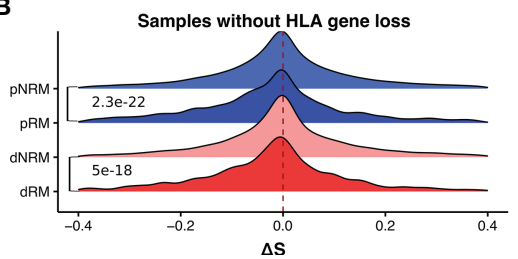

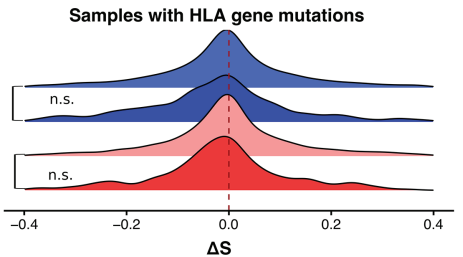

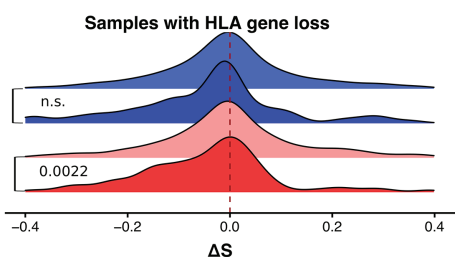

FIGURE 4 | Difference in MHC-I binding affinities of somatic mutations in melanoma samples with and without HLA gene deficiency. (A) The distribution of $\triangle$ S of somatic mutations in samples with and without HLA gene mutations. (B) The distribution of $\Delta$ S of somatic mutations in samples with and without HLA gene loss. $\mathrm{dRM}$, recurrent mutation in driver genes; dNRM, non-recurrent in driver genes; pRM, recurrent mutation in passenger genes; pNRM, non-recurrent in passenger genes. $P_{a d j}$-values were calculated using two-sided Wilcoxon rank-sum tests and adjusted for multiple testing by the Holm-Bonferroni method. n.s., not significant.

scores were also significantly lower in melanoma samples with higher levels of recurrent mutations (Figure 5B). These results suggest a differential tumor microenvironment between patients with high and low numbers of recurrent mutations sample groups.

\section{MHC-I Binding Affinities of Detected SKCM Mutations and Their Possible Alterations}

In this study, the binding affinity of peptides with MHC-I complex was calculated based on the HLA genotype of the patient. For each non-synonymous somatic mutation, we also calculated MHC-I binding scores using HLA genotypes of patients that did not carry the somatic mutation (i.e., non-self HLA genotype). Comparative analyses identified a total of 46 somatic mutations that showed significantly lower $\Delta \mathrm{S}$ with self-HLA than with non-self HLA genotypes (Table 1, P-value $\leq 0.001$, two-sided Wilcoxon rank-sum test). We hypothesize that his set of mutations has been shaped by immune selection in a patient-specific manner.

Next, we examined whether the observed amino acid change for a given somatic mutation tended to have a different MHC-I

A
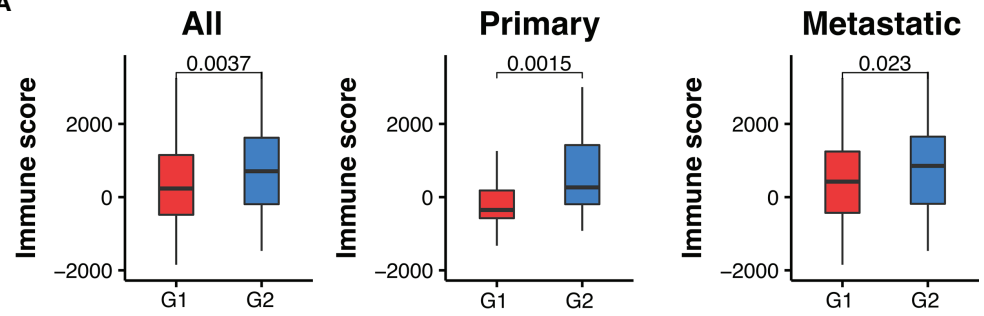

B

All

Primary
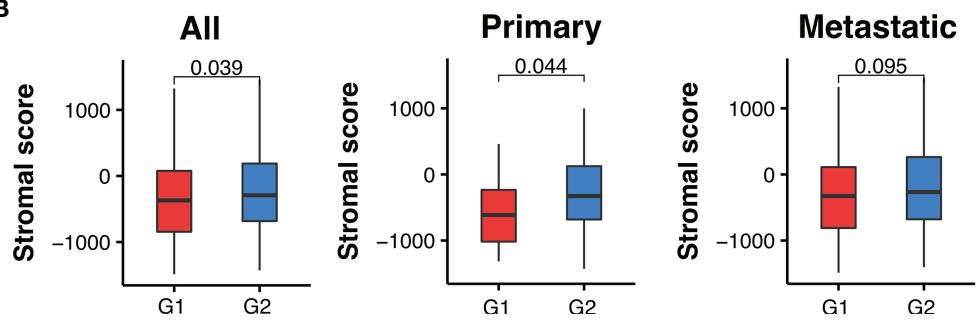

FIGURE 5 | Melanoma samples with $\geq 7$ recurrent mutations (G1) have lower immune and stromal scores than those with $<7$ (G2). (A) Comparison of immune scores between recurrent and non-recurrent mutations in all, primary and metastatic melanoma samples. (B) Comparison of stromal scores between recurrent and

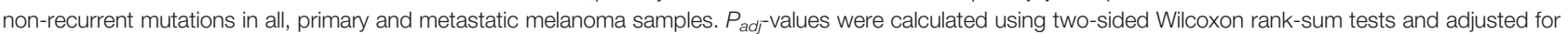
multiple testing by the Holm-Bonferroni method. 
TABLE 1 | The mutations, which have significant lower $\Delta S$ in own patient's HLA alleles than non-self patients' HLA alleles, were identified in SKCM (P-value $\leq 0.001$, two-sided Wilcoxon rank-sum test).

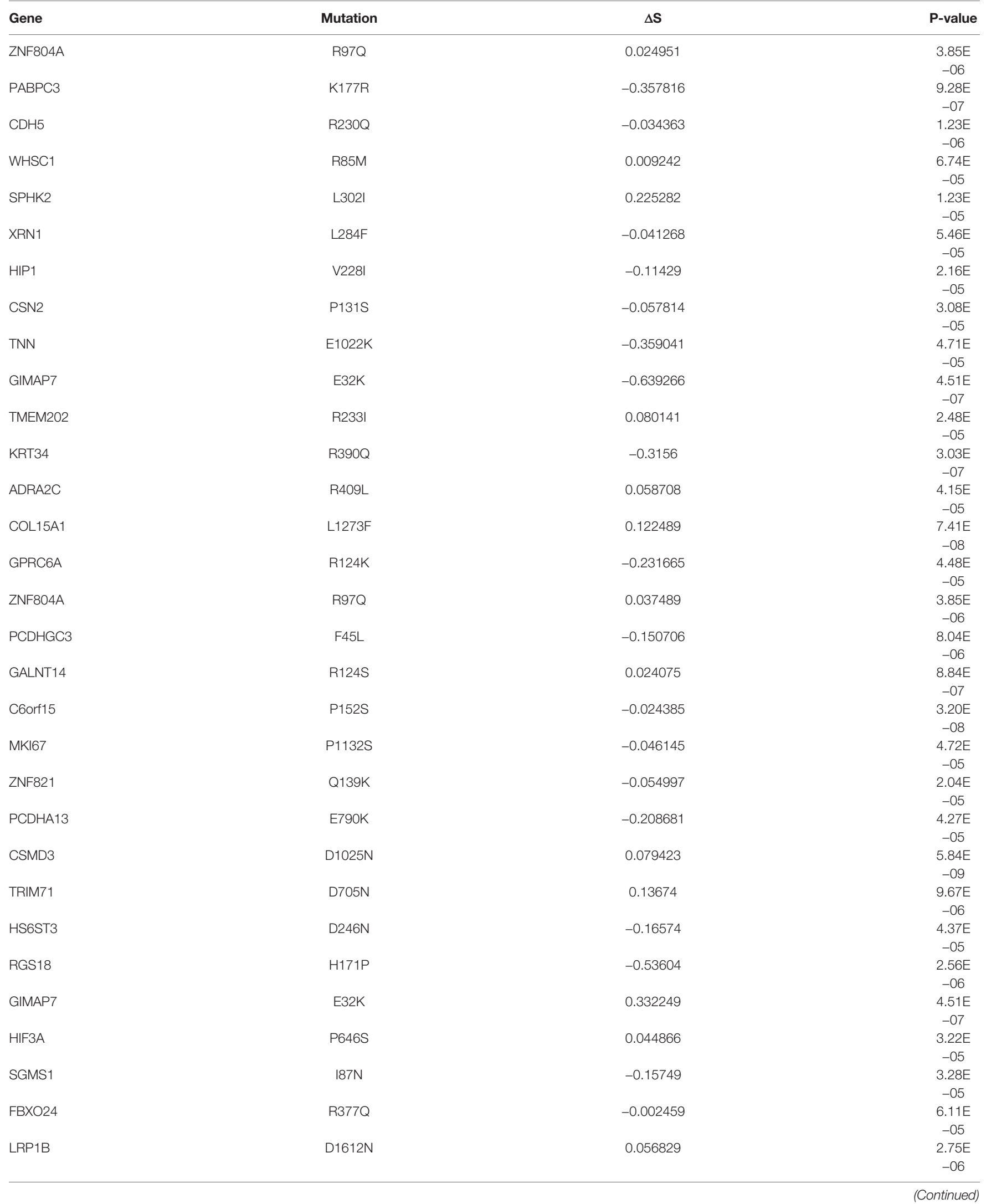


TABLE 1 | Continued

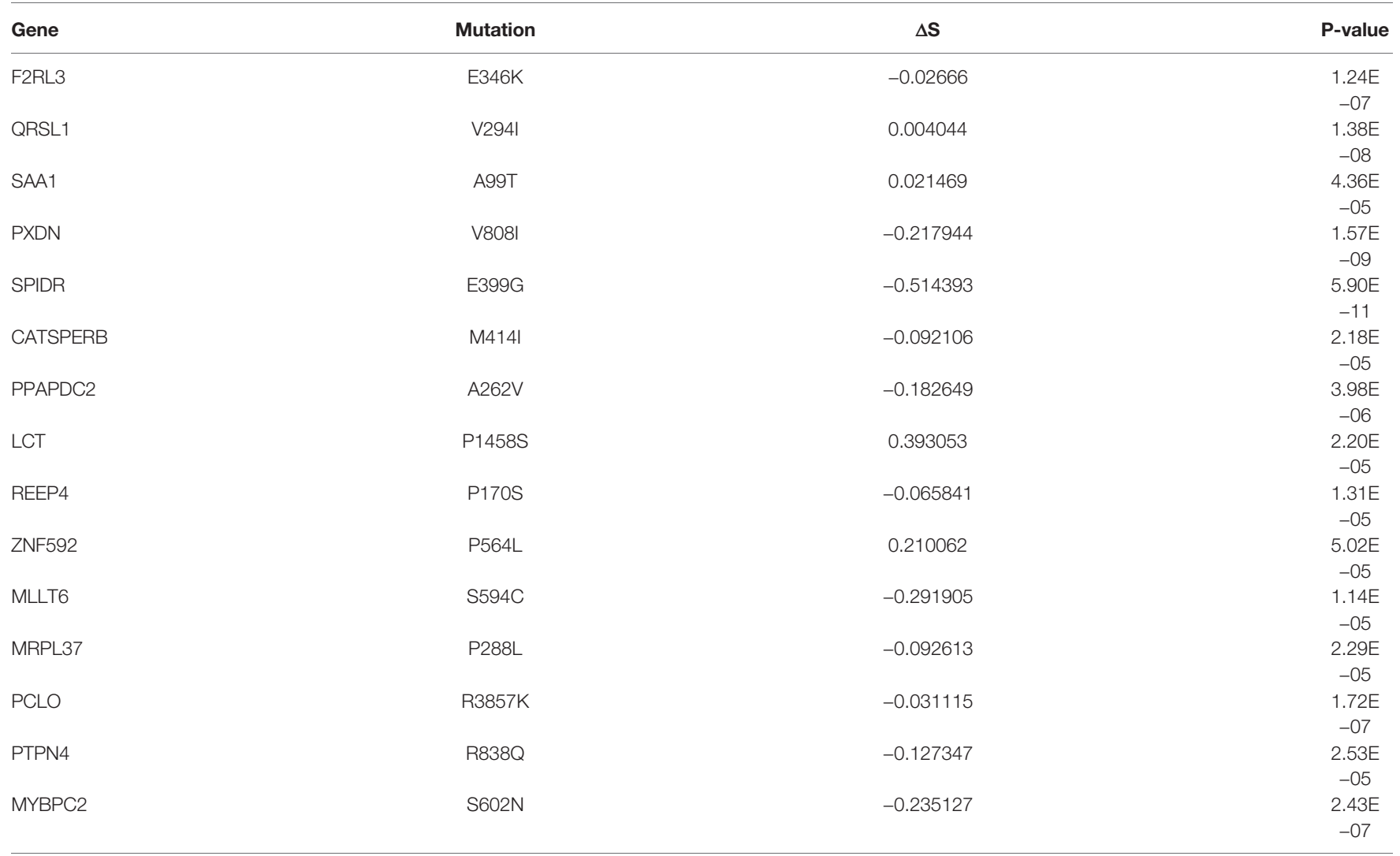

binding affinity compared with the other 18 possible amino acid changes. As shown in Figure 6A, our result indicated that the observed amino acid change tended to be lower binding affinity than other possible changes. This trend is more obvious when comparing $\Delta S$ for the observed and other amino acid changes (Figure 6B). Indeed, the $\Delta S$ values for the observed changes were significantly lower than those for the other possible amino acid changes $\left(P_{a d j}=6.9 \mathrm{e}-29\right.$, ANOVA test and adjusted for multiple testing by the Benjamini-Hochberg method).

\section{DISCUSSION}

In this study, we demonstrated that $\mathrm{T}$ cell-mediated immunosurveillance likely contributes to the mutational landscape in SKCM tumors. Here we used the MHC-I binding difference between mutation-derived neoantigens $\left(S_{m u}\right)$ and their corresponding wild-type peptides $\left(S_{w t}\right)$ to measure the effect of mutations on antigenicity, i.e., $\Delta S=S_{m u}-S_{w t}$. This value has been defined as differential aggretope index (DAI) in previous analysis by Duan et al. (8). Following that, DAI has been used in several studies $(8,9,37-40)$ to measure the antigenicity of neoantigens, using the same formula or a variant version (e.g., $S_{m u} / S_{w t}$ ) (39). DAI has been used to select the best potential immunoprotective neoepitopes (i.e., those with the highest DAI values) from a vast number of somatic mutations (8). Sedlacek et al. reported that in the absence of CD91 on dendritic cells there was a rise of neoepitopes with high DAI, suggesting the function CD91 during immunosurveillance (40). In this study, by showing the $\Delta \mathrm{S}$ difference between recurrent and non-recurrent somatic mutation derived neoantigens, we showed the potential effect of immunosurveillance on shaping the somatic mutation landscape. In addition, we have used another DAI metric $\left(S_{m u} / S_{w t}\right)$ to compare recurrent and non-recurrent mutations, and observed consistent results as $\Delta \mathrm{S}$ (Figure $\mathbf{S 3}$ ).

In this study, we defined recurrent somatic mutations as those occurring in at least three melanoma patients. To evaluate the robustness of our analysis, we have also applied different thresholds (the number of patients with a specific somatic mutation) to define recurrent mutations. Specifically, we defined recurrent mutations as those presenting in $\geq s$ ( $s=3,4$, $5,6,7)$ different melanoma patients, and compared with nonrecurrent mutations. We observed significant lower $\Delta S$ for recurrent mutations regardless of threshold setting (Figure S4).

Our analysis indicated that neoantigens derived from recurrent mutations tended to have lower binding affinity with the MHC-I complex compared to those from non-recurrent mutations. We found that in both driver and passenger genes, recurrent mutations tended to have reduced MHC-I binding affinities compared to non-recurrent mutations (Figure 3 and Figure S2). These results support the model (Figure 7) in which tumor cells presenting neoantigens with strong MHC-I binding capacity are more likely to be eliminated during tumorigenesis and are less likely to be observed (i.e., non-recurrent) at the 


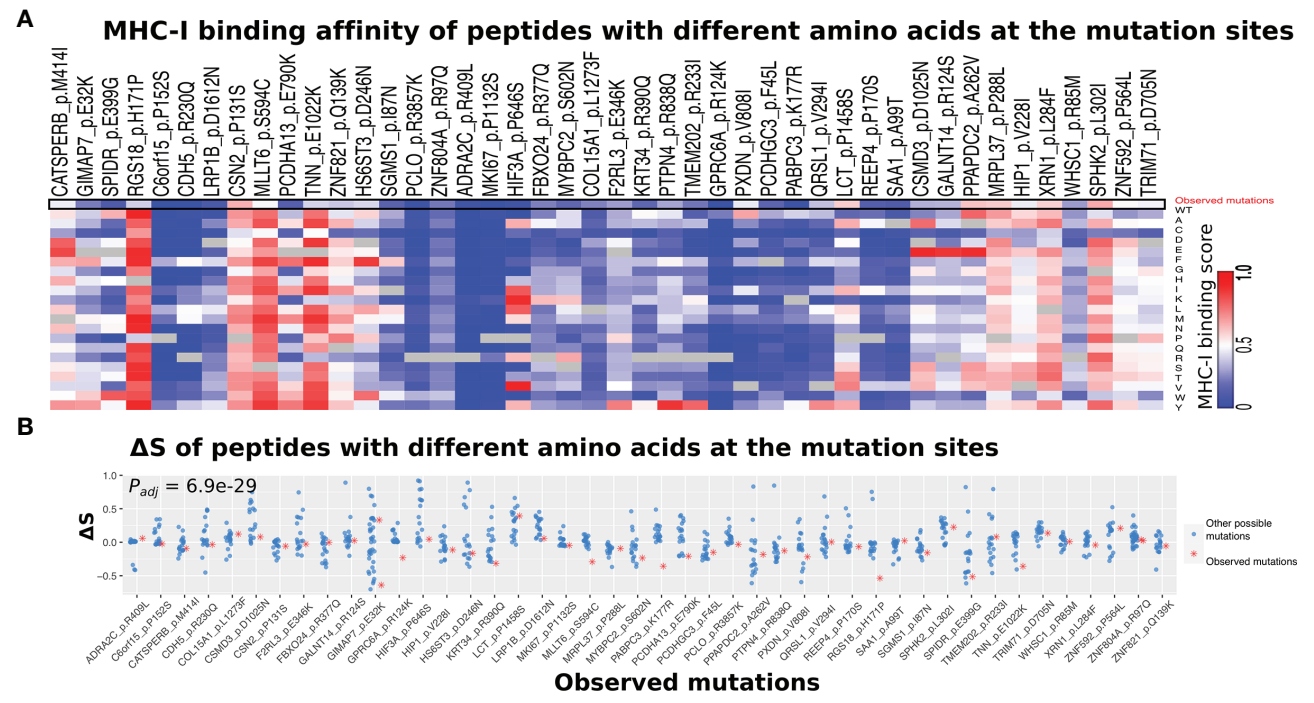

FIGURE 6 | Comparison of MHC-I binding alterations between observed and other possible amino acid changes. (A) Heatmap showing a list of 46 somatic mutations that derive neoantigen with significantly lower $\Delta S$ using the self HLA genotype than non-self HLA genotypes. The HLA genotypes of melanoma patients that do not have a specific mutation were selected as non-self. (B) The observed amino acid changes (red dots) tend to have lower $\Delta \mathrm{S}$ than those unobserved ones (other 18 possible amino acid changes) at the same position. The $P_{\text {adj }}$-value was calculated by the ANOVA test and adjusted for multiple testing by the BenjaminiHochberg method.

population level. In contrast, somatic mutations that result in neoantigens with low MHC-I binding affinity are under less selective pressure by the host immune system and are therefore more likely to be observed in tumors. This model is consistent with observations from previous studies (41-45).

\section{CONCLUSION}

Neoantigens derived from recurrent mutations generally have low binding affinities with MHC-I complex. Based on this observation and previous studies, we proposed a model to explain how $\mathrm{T}$ cell-mediated immunosurveillance shapes the mutational landscape in tumors.

\section{MATERIALS AND METHODS}

\section{Data Preparation and Processing}

MuTect2-called whole-exome sequencing (WES) mutation annotation format (MAF) files of the SKCM from The Cancer Genome Atlas (TCGA) were downloaded from the Genomic Data Commons (GDC) data portal (https://portal.gdc.cancer. gov; data release v7). From these files, we selected all nonsynonymous mutations for downstream analysis.

The TCGA level 3 gene expression data of melanoma were obtained from the Genomic Data Commons (GDC, available at: https://portal.gdc.cancer.gov/) Data Portal on Mar 7, 2019. From the GDC, we downloaded the expression profiles for tumors with the disease type "melanoma" from the "TCGA-SKCM".
Fragments per kilobase of exon per million reads mapped (FPKM) were used for expression quantification for a total of 20,501 protein-coding genes annotated in the TCGA data portal.

\section{MHC-I Binding Affinity Predictions}

HLA affinities of mutated and matched-wild-type peptides were predicted for their sample-specific HLA genotype (a specific combination of two HLA-A, two HLA-B, and two HLA-C alleles) using NetMHCpan4.0 (25). Firstly, for each type of HLA allele (HLA-A, HLA-B, and HLA-C), many subtypes exist and most of these HLA allele subtypes account for a very small proportion of the population $(\leq 0.01 \%)$. To ensure stability and avoid over-calculation, all HLA allele subtypes with a proportion greater than $0.01 \%$ were selected in this study. A total of 92 HLA alleles were selected for the peptide binding HLA allele prediction. Thirty subtypes of HLA-A alleles cover $99.76 \%$ of the population; 39 subtypes of HLA-B alleles cover $99.08 \%$ of the population; 23 subtypes of HLA-C alleles cover $99.81 \%$ of the population. The derived frequencies for each HLA allele were compared with the allele frequencies from a healthy US blood donor population, downloaded from the Allele frequency net (46), Supplementary Table 3). Second, each mutated and matched wild-type peptide was predicted for the 92 HLA alleles. Finally, the patient-specific MHC-I binding affinities of each mutated and matched wild-type peptides were obtained.

\section{Calculation of Patient-Specific MHC-I Binding Affinity of Somatic Mutations}

We used an MHC-I binding score to represent the binding affinity of a non-synonymous mutation with patient-specific 


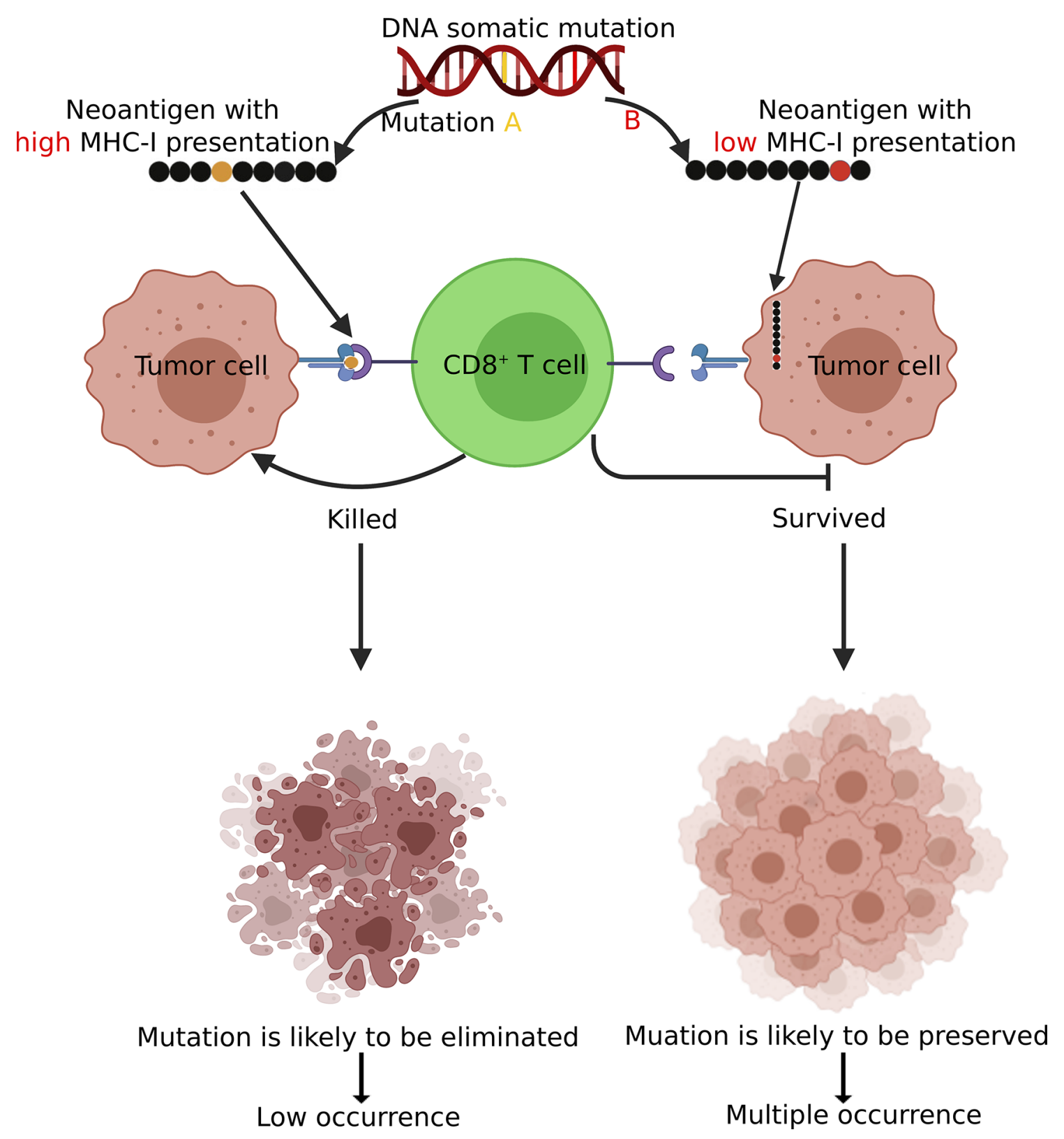

FIGURE 7 | A schematic diagram demonstrating how immunosurveillance shapes the somatic mutation landscape in tumors. Somatic mutations leading to CD8+ neoantigen with high MHC-I binding affinity are more likely to be recognized and eliminated by $\mathrm{T}$ cells and there tend to have low occurrences (non-recurrent) in tumor. In contrast, mutations with low MHC-I binding affinity are more likely to be preserved and present in multiple patients. Figure created using BioRender (https:// biorender.com/).

HLA class I alleles (two HLA-A, two HLA-B, and two HLA-C) (Figure 1). First, for each non-synonymous mutation, we enumerated the nine 9-mers (peptides with 9 amino acids) that overlap with the mutated residue and applied NetMHCPan4.0 (25) to calculate their binding affinity with the MHC-I complex using patient-specific HLA class I alleles (two HLA-A, two HLA$B$, and two HLA-C). Second, we calculated the maximum value for each HLA allele to capture the peptide with the highest binding affinity, resulting in six HLA allele-specific affinity scores for each mutation. Finally, we selected the maximum value to represent the best presentation potential by the MHC-I complex for peptides derived from a specific non-synonymous mutation. The HLA typing of all the TCGA-SKCM samples was downloaded from the TCIA (ref link: https://tcia.at/home).

To measure the effect of somatic mutations on antigenicity, the MHC-I binding difference between mutation-derived neoantigens $\left(S_{m u}\right)$ and their corresponding wild-type peptides $\left(S_{w t}\right)$ was calculated, denoted by $\Delta \mathrm{S}=S_{m u}-S_{w t}$. This definition is 
consistent with the differential aggretope index (DAI) introduced in the previous studies by Duan et al. $(8,9,40)$.

\section{Evaluation of the MHC-I Binding Prediction Pipeline}

To test the performance of affinity scores representing actual MHC class I presentation, we downloaded independent mass spectrum data of 5 different human cell lines (Fibroblast, SupB15W, JY, HCC1937, and HCC1143) from BassaniSternberg et al. (27). These peptides were observed in complex with MHC-I on the cell surface across known HLA alleles. An independent non-MHC-binding peptide dataset was downloaded from Abelin et al.; these peptides were validated to not bind with known MHC-I alleles in mass spectrometry experiments (26). Detailed information on the classification of MHC-binding peptides and independent non-MHC-binding peptide controls are shown in Supplementary Tables 1A-G.

\section{Estimation of Stromal and Immune Scores}

Sample-specific immune scores and stromal scores were calculated based on gene expression profiles (described above). The ESTIMATE algorithm (36) was applied to the normalized expression matrix for estimating the stromal and immune scores for each melanoma sample.

\section{Statistical Analysis}

The $\mathrm{R}$ statistical package was used for all data processing and statistical analysis ( $\mathrm{R}$ package: stats v3.6.2). All details of the statistical tests are specified in the associated text or figure legends. For the comparison of the observed mutations and their according other possible mutations, the $\mathrm{P}$-value was calculated by using an ANOVA test and adjusted for multiple testing by the Benjamini-Hochberg method. For the other statistical analyses, Pvalues were calculated by using the "Wilcox_test" function from the R package: stats v3.6.2, which applies the two-sided Wilcoxon ranksum test and corrected multiple testing using the Holm-Bonferroni method. A statistically significant difference was assumed when adjusted $\mathrm{P} \leq 0.05$.

\section{REFERENCES}

1. Teng MW, Kershaw MH, Smyth MJ. Cancer Immunoediting: From Surveillance to Escape. Cancer Immunother (Elsevier) (2013) 3:85-99. doi: 10.1016/B978-0-12-394296-8.00007-5

2. Burnet F. The Concept of Immunological Surveillance. Immunol Aspects Neoplasia (1970) 13:1-27. doi: 10.1159/000386035

3. Reeves E, James E. Antigen Processing and Immune Regulation in the Response to Tumours. Immunology (2017) 150:16-24. doi: 10.1111/ imm. 12675

4. Ribatti D. The Concept of Immune Surveillance Against Tumors: The First Theories. Oncotarget (2017) 8:7175. doi: 10.18632/oncotarget.12739

5. Traba J, Sack MN, Waldmann TA, Anton OM. Immunometabolism at the Nexus of Cancer Therapeutic Efficacy and Resistance. Front Immunol (2021) 12. doi: 10.3389/fimmu.2021.657293

6. Vitale I, Sistigu A, Manic G, Rudqvist NP, Trajanoski Z, Galluzzi L. Mutational and Antigenic Landscape in Tumor Progression and Cancer Immunotherapy. Trends Cell Biol (2019) 29:396-416. doi: 10.1016/ j.tcb.2019.01.003

\section{DATA AVAILABILITY STATEMENT}

The original contributions presented in the study are included in the article/Supplementary Material. Further inquiries can be directed to the corresponding author.

\section{AUTHOR CONTRIBUTIONS}

CC conceived the project. CJ, HW, and CC performed computational analyses. CJ, CC, ES, and $\mathrm{KZ}$ wrote the manuscript. CJ, C-CC, ES, and CC interpreted the results. CC supervised the project. All authors contributed to the article and approved the submitted version.

\section{FUNDING}

This work is supported by the Cancer Prevention Research Institute of Texas (CPRIT; RR180061, to CC) and the NCI of the NIH (1R21CA227996, to CC), and the T32 training grant of the NIH (T32 AI007363, to ES). CC is a CPRIT Scholar in Cancer Research.

\section{ACKNOWLEDGMENTS}

We would like to thank all members of the Cheng lab for their valuable discussions and critical feedback. We especially thank Xiuying Li and Yadong Dong for the valuable suggestions.

\section{SUPPLEMENTARY MATERIAL}

The Supplementary Material for this article can be found online at: https://www.frontiersin.org/articles/10.3389/fimmu.2021. 703821/full\#supplementary-material

7. Danilova L, Anagnostou V, Caushi JX, Sidhom JW, Guo H, Chan HY, et al. The Mutation-Associated Neoantigen Functional Expansion of Specific T Cells (Manafest) Assay: A Sensitive Platform for Monitoring Antitumor Immunity. Cancer Immunol Res (2018) 6:888-99. doi: 10.1158/2326-6066.CIR-18-0129

8. Duan F, Duitama J, Al Seesi S, Ayres CM, Corcelli SA, Pawashe AP, et al. Genomic and Bioinformatic Profiling of Mutational Neoepitopes Reveals New Rules to Predict Anticancer Immunogenicity. J Exp Med (2014) 211:2231-48. doi: 10.1084/jem.20141308

9. Ghorani E, Rosenthal R, McGranahan N, Reading J, Lynch M, Peggs K, et al. Differential Binding Affinity of Mutated Peptides for Mhc Class I is a Predictor of Survival in Advanced Lung Cancer and Melanoma. Ann Oncol (2018) 29:271-9. doi: 10.1093/annonc/mdx687

10. Wang RF, Wang HY. Immune Targets and Neoantigens for Cancer Immunotherapy and Precision Medicine. Cell Res (2017) 27:11-37. doi: $10.1038 / \mathrm{cr} .2016 .155$

11. Castro A, Ozturk K, Pyke RM, Xian S, Zanetti M, Carter H. Elevated Neoantigen Levels in Tumors With Somatic Mutations in the Hla-a, Hla-B, Hla-C and B2m Genes. BMC Med Genomics (2019) 12:1-13. doi: 10.1186/ s12920-019-0544-1 
12. Chandrashekar P, Ahmadinejad N, Wang J, Sekulic A, Egan JB, Asmann YW, et al. Somatic Selection Distinguishes Oncogenes and Tumor Suppressor Genes. Bioinformatics (2020) 36:1712-7. doi: 10.1093/bioinformatics/btz851

13. Martincorena I, Raine KM, Gerstung M, Dawson KJ, Haase K, Van Loo P, et al. Universal Patterns of Selection in Cancer and Somatic Tissues. Cell (2017) 171:1029-41. doi: 10.1016/j.cell.2017.09.042

14. Rosenthal R, Cadieux EL, Salgado R, Al Bakir M, Moore DA, Hiley CT, et al. Neoantigen-Directed Immune Escape in Lung Cancer Evolution. Nature (2019) 567:479-85. doi: 10.1038/s41586-019-1032-7

15. Zapata L, Pich O, Serrano L, Kondrashov FA, Ossowski S, Schaefer MH. Negative Selection in Tumor Genome Evolution Acts on Essential Cellular Functions and the Immunopeptidome. Genome Biol (2018) 19:1-17. doi: 10.1186/s13059-018-1434-0

16. Rooney MS, Shukla SA, Wu CJ, Getz G, Hacohen N. Molecular and Genetic Properties of Tumors Associated With Local Immune Cytolytic Activity. Cell (2015) 160:48-61. doi: 10.1016/j.cell.2014.12.033

17. Schreiber RD, Old LJ, Smyth MJ. Cancer Immunoediting: Integrating Immunity's Roles in Cancer Suppression and Promotion. Science (2011) 331:1565-70. doi: 10.1126/science.1203486

18. Claeys A, Luijts T, Marchal K, Van den Eynden J. Low Immunogenicity of Common Cancer Hot Spot Mutations Resulting in False Immunogenic Selection Signals. PloS Genet (2021) 17:e1009368. doi: 10.1371/journal. pgen. 1009368

19. Van den Eynden J, Jiménez-Sánchez A, Miller ML, Larsson E. Lack of Detectable Neoantigen Depletion Signals in the Untreated Cancer Genome. Nat Genet (2019) 51:1741-8. doi: 10.1038/s41588-019-0532-6

20. Dentro SC, Leshchiner I, Haase K, Tarabichi M, Wintersinger J, Deshwar AG, et al. Characterizing Genetic Intra-Tumor Heterogeneity Across 2,658 Human Cancer Genomes. Cell (2021) 184:2239-54.e39. doi: 10.1016/j.cell.2021.03.009

21. Kumar S, Warrell J, Li S, McGillivray PD, Meyerson W, Salichos L, et al. Passenger Mutations in More Than 2,500 Cancer Genomes: Overall Molecular Functional Impact and Consequences. Cell (2020) 180:915-27. doi: 10.1016/j.cell.2020.01.032

22. McFarland CD, Korolev KS, Kryukov GV, Sunyaev SR, Mirny LA. Impact of Deleterious Passenger Mutations on Cancer Progression. Proc Natl Acad Sci (2013) 110:2910-5. doi: 10.1073/pnas.1213968110

23. Yang F, Kim DK, Nakagawa H, Hayashi S, Imoto S, Stein L, et al. Quantifying Immune-Based Counterselection of Somatic Mutations. PloS Genet (2019) 15: e1008227. doi: 10.1371/journal.pgen.1008227

24. McGranahan N, Rosenthal R, Hiley CT, Rowan AJ, Watkins TB, Wilson GA, et al. Allele-Specific Hla Loss and Immune Escape in Lung Cancer Evolution. Cell (2017) 171:1259-71. doi: 10.1016/j.cell.2017.10.001

25. Jurtz V, Paul S, Andreatta M, Marcatili P, Peters B, Nielsen M. Netmhcpan4.0: Improved Peptide-Mhc Class I Interaction Predictions Integrating Eluted Ligand and Peptide Binding Affinity Data. J Immunol (2017) 199:3360-8. doi: 10.4049/jimmunol.1700893

26. Abelin JG, Keskin DB, Sarkizova S, Hartigan CR, Zhang W, Sidney J, et al. Mass Spectrometry Profiling of Hla-Associated Peptidomes in Mono-Allelic Cells Enables More Accurate Epitope Prediction. Immunity (2017) 46:315-26. doi: 10.1016/j.immuni.2017.02.007

27. Bassani-Sternberg M, Pletscher-Frankild S, Jensen LJ, Mann M. Mass Spectrometry of Human Leukocyte Antigen Class I Peptidomes Reveals Strong Effects of Protein Abundance and Turnover on Antigen Presentation. Mol Cell Proteomics (2015) 14:658-73. doi: 10.1074/mcp.M114.042812

28. Hendrickx W, Simeone I, Anjum S, Mokrab Y, Bertucci F, Finetti P, et al. Identification of Genetic Determinants of Breast Cancer Immune Phenotypes by Integrative Genome-Scale Analysis. Oncoimmunology (2017) 6:e1253654. doi: 10.1080/2162402X.2016.1253654

29. Marty R, Kaabinejadian S, Rossell D, Slifker MJ, van de Haar J, Engin HB, et al. Mhc-I Genotype Restricts the Oncogenic Mutational Landscape. Cell (2017) 171:1272-83. doi: 10.1016/j.cell.2017.09.050

30. Roudko V, Bozkus CC, Orfanelli T, McClain CB, Carr C, O’Donnell T, et al. Shared Immunogenic Poly-Epitope Frameshift Mutations in Microsatellite Unstable Tumors. Cell (2020) 183:1634-49. doi: 10.1016/j.cell.2020.11.004

31. Vogelstein B, Papadopoulos N, Velculescu VE, Zhou S, Diaz LA, Kinzler KW. Cancer Genome Landscapes. science (2013) 339:1546-58. doi: 10.1126/science.1235122

32. Lu YC, Robbins PF. Targeting Neoantigens for Cancer Immunotherapy. Int Immunol (2016) 28:365-70. doi: 10.1093/intimm/dxw026
33. Chung IF, Chen CY, Su SC, Li CY, Wu KJ, Wang HW, et al. Driverdbv2: A Database for Human Cancer Driver Gene Research. Nucleic Acids Res (2016) 44:D975-9. doi: 10.1093/nar/gkv1314

34. Perea F, Sánchez-Palencia A, Gómez-Morales M, Bernal M, Concha Á, García MM, et al. Hla Class I Loss and Pd-L1 Expression in Lung Cancer: Impact on T-Cell Infiltration and Immune Escape. Oncotarget (2018) 9:4120. doi: 10.18632/oncotarget.23469

35. Taylor AM, Shih J, Ha G, Gao GF, Zhang X, Berger AC, et al. Genomic and Functional Approaches to Understanding Cancer Aneuploidy. Cancer Cell (2018) 33:676-89. doi: 10.1016/j.ccell.2018.03.007

36. Yoshihara K, Shahmoradgoli M, Martínez E, Vegesna R, Kim H, TorresGarcia W, et al. Inferring Tumour Purity and Stromal and Immune Cell Admixture From Expression Data. Nat Commun (2013) 4:1-11. doi: 10.1038/ ncomms 3612

37. Brennick CA, George MM, Moussa MM, Hagymasi AT, Al Seesi S, Shcheglova TV, et al. An Unbiased Approach to Defining Bona Fide Cancer Neoepitopes That Elicit Immune-Mediated Cancer Rejection. J Clin Invest (2021) 131:27687. doi: 10.1172/JCI142823

38. Ebrahimi-Nik H, Michaux J, Corwin WL, Keller GL, Shcheglova T, Pak H, et al. Mass Spectrometry-Driven Exploration Reveals Nuances of Neoepitope-Driven Tumor Rejection. JCI Insight (2019) 4:1-15. doi: 10.1172/jci.insight.129152

39. Rech AJ, Balli D, Mantero A, Ishwaran H, Nathanson KL, Stanger BZ, et al. Tumor Immunity and Survival as a Function of Alternative Neopeptides in Human Cancer. Cancer Immunol Res (2018) 6:276-87. doi: 10.1158/2326-6066.CIR-17-0559

40. Sedlacek AL, Younker TP, Zhou YJ, Borghesi L, Shcheglova T, Mandoiu II, et al. Cd91 on Dendritic Cells Governs Immunosurveillance of Nascent, Emerging Tumors. JCI Insight (2019) 4:1-11. doi: 10.1172/jci.insight.127239

41. Ballhausen A, Przybilla MJ, Jendrusch M, Haupt S, Pfaffendorf E, Seidler F, et al. The Shared Frameshift Mutation Landscape of Microsatellite-Unstable Cancers Suggests Immunoediting During Tumor Evolution. Nat Commun (2020) 11:1-13. doi: 10.1038/s41467-020-18514-5

42. Fujimoto A, Furuta M, Totoki Y, Tsunoda T, Kato M, Shiraishi Y, et al. WholeGenome Mutational Landscape and Characterization of Noncoding and Structural Mutations in Liver Cancer. Nat Genet (2016) 48:500-9. doi: 10.1038/ng.3547

43. Gee MH, Han A, Lofgren SM, Beausang JF, Mendoza JL, Birnbaum ME, et al. Antigen Identification for Orphan T Cell Receptors Expressed on TumorInfiltrating Lymphocytes. Cell (2018) 172:549-63. doi: 10.1016/j.cell.2017.11.043

44. Hayward NK, Wilmott JS, Waddell N, Johansson PA, Field MA, Nones K, et al. Whole-Genome Landscapes of Major Melanoma Subtypes. Nature (2017) 545:175-80. doi: 10.1038/nature22071

45. Wu J, Zhao W, Zhou B, Su Z, Gu X, Zhou Z, et al. Tsnadb: A Database for Tumor-Specific Neoantigens From Immunogenomics Data Analysis. Genomics Proteomics Bioinf (2018) 16:276-82. doi: 10.1016/j.gpb.2018.06.003

46. González-Galarza FF, Takeshita LY, Santos EJ, Kempson F, Maia MHT, ALSd S, et al. Allele Frequency Net 2015 Update: New Features for Hla Epitopes, Kir and Disease and Hla Adverse Drug Reaction Associations. Nucleic Acids Res (2015) 43:D784-8. doi: 10.1093/nar/gku1166

Conflict of Interest: Author C-CC was employed by the company Chempartner Corporation.

The remaining authors declare that the research was conducted in the absence of any commercial or financial relationships that could be construed as a potential conflict of interest.

Publisher's Note: All claims expressed in this article are solely those of the authors and do not necessarily represent those of their affiliated organizations, or those of the publisher, the editors and the reviewers. Any product that may be evaluated in this article, or claim that may be made by its manufacturer, is not guaranteed or endorsed by the publisher.

Copyright (๑ 2022 Jiang, Schaafsma, Hong, Zhao, Zhu, Chao and Cheng. This is an open-access article distributed under the terms of the Creative Commons Attribution License (CC BY). The use, distribution or reproduction in other forums is permitted, provided the original author(s) and the copyright owner(s) are credited and that the original publication in this journal is cited, in accordance with accepted academic practice. No use, distribution or reproduction is permitted which does not comply with these terms. 